ELECTRONIC RESEARCH ANNOUNCEMENTS OF THE AMERICAN MATHEMATICAL SOCIETY

Volume 10, Pages 11-20 (March 4, 2004)

S $1079-6762(04) 00125-8$

\title{
ENDOSCOPIC DECOMPOSITION OF CHARACTERS OF CERTAIN CUSPIDAL REPRESENTATIONS
}

\author{
DAVID KAZHDAN AND YAKOV VARSHAVSKY
}

(Communicated by Svetlana Katok)

\begin{abstract}
We construct an endoscopic decomposition for local $L$-packets associated to irreducible cuspidal Deligne-Lusztig representations. Moreover, the obtained decomposition is compatible with inner twistings.
\end{abstract}

\section{INTRODUCTION}

Let $E$ be a local non-Archimedean field, with ring of integers $\mathcal{O}$ and residue field $\mathbb{F}_{q}$ of characteristic $p$. We denote by $\Gamma \supset W \supset I$ the absolute Galois, the Weil, and the inertia groups of $E$. Let $G$ be a reductive group over $E,{ }^{L} G=\widehat{G} \rtimes W$ its complex Langlands dual group, and $\mathcal{D}(G(E))$ the space of invariant distributions on $G(E)$.

Every admissible homomorphism $\lambda: W \rightarrow{ }^{L} G$ (see [Ko1, $\left.\S 10\right]$ ) gives rise to a finite group $S_{\lambda}:=\pi_{0}\left(Z_{\widehat{G}}(\lambda) / Z(\widehat{G})^{\Gamma}\right)$, where $Z_{\widehat{G}}(\lambda)$ is the centralizer of $\lambda(W)$ in $\widehat{G}$. Every conjugacy class $\kappa$ of $S_{\lambda}$ defines an endoscopic subspace $\mathcal{D}_{\kappa, \lambda}(G(E)) \subset$ $\mathcal{D}(G(E))$. For simplicity, we will restrict ourselves to the elliptic case, where $\lambda(W)$ does not lie in any proper Levi subgroup of ${ }^{L} G$.

Langlands conjectured that every elliptic $\lambda$ corresponds to a finite set $\Pi_{\lambda}$, called an $L$-packet, of cuspidal irreducible representations of $G(E)$. Moreover, the subspace $\mathcal{D}_{\lambda}(G(E)) \subset \mathcal{D}(G(E))$, generated by the characters $\{\chi(\pi)\}_{\pi \in \Pi_{\lambda}}$, must have an endoscopic decomposition. More precisely, it is expected ([La1, IV, 2]) that there exists a basis $\left\{a_{\pi}\right\}_{\pi \in \Pi_{\lambda}}$ of the space of central functions on $S_{\lambda}$ such that $\chi_{\kappa, \lambda}:=\sum_{\pi \in \Pi_{\lambda}} a_{\pi}(\kappa) \chi(\pi)$ belongs to $\mathcal{D}_{\kappa, \lambda}(G)$ for every conjugacy class $\kappa$ of $S_{\lambda}$.

The goal of this paper is to construct the endoscopic decomposition of $\mathcal{D}_{\lambda}(G(E))$ for tamely ramified $\lambda$ 's such that $Z_{\widehat{G}}(\lambda(I))$ is a maximal torus. In this case, $G$ splits over an unramified extension of $E$, and $\lambda$ factors through ${ }^{L} T \hookrightarrow{ }^{L} G$ for an elliptic unramified maximal torus $T$ of $G$. By the local Langlands correspondence for tori ([L2] $)$, a homomorphism $\lambda: W \rightarrow{ }^{L} T$ defines a tamely ramified homomorphism $\theta: T(E) \rightarrow \mathbb{C}^{\times}$. Each $\kappa \in S_{\lambda}=\widehat{T}^{\Gamma} / Z(\widehat{G})^{\Gamma}$ gives rise to an elliptic endoscopic datum $\mathcal{E}_{\kappa, \lambda}$ of $G$, while the characters of $S_{\lambda}$ are in bijection with the conjugacy classes of embeddings $T \hookrightarrow G$, stably conjugate to the inclusion. Therefore each

Received by the editors September 18, 2003 and, in revised form, January 19, 2004.

2000 Mathematics Subject Classification. Primary 22E50; Secondary 22E35.

Key words and phrases. Endoscopy, Deligne-Lusztig representations.

The work of the second author was supported by the Israel Science Foundation (Grant No. 38/01-1). 
character $a$ of $S_{\lambda}$ gives rise to an irreducible cuspidal representation $\pi_{a, \lambda}$ of $G(E)$ (denoted by $\pi_{a, \theta}$ in Notation [2.3).

Our main result asserts, for fields $E$ of sufficiently large residual characteristic, that each $\chi_{\kappa, \lambda}:=\sum_{a} a(\kappa) \chi\left(\pi_{a, \lambda}\right)$ is $\mathcal{E}_{\kappa, \lambda}$-stable. Moreover, the resulting endoscopic decomposition of $\mathcal{D}_{\lambda}(G(E))$ is compatible with inner twistings. For simplicity, we restrict ourselves to local fields of characteristic zero, while the case of positive characteristic follows by approximation (see [Ka3], De] ).

Our argument goes as follows. First we prove the stability of the restriction of $\chi_{\kappa, \lambda}$ to the subset of topologically unipotent elements of $G(E)$. If $p$ is sufficiently large, this assertion reduces to the analogous assertion about distributions on the Lie algebra. Now the stability follows from a combination of a Springer hypothesis Ka1 and a generalization of a theorem of Waldspurger [Wa. To prove the result in general, we use the topological Jordan decomposition ([Ka2]).

When this work was in the process of writing, we have heard that S. DeBacker and M. Reeder obtained similar results.

Notation and conventions. In addition to the notation introduced above, we use the following conventions:

For a reductive group $G$, always assumed to be connected, we denote by $Z(G)$, $G^{\text {ad }}, G^{\text {der }}, G^{\text {sc }}, G_{\delta}$, and $G^{\text {sr }}$ the center of $G$, the adjoint group of $G$, the derived group of $G$, the simply connected covering of $G^{\text {der }}$, the centralizer of $\delta \in G$, and the set of strongly regular semisimple elements of $G$ (that is, the set of $\delta \in G$ such that $G_{\delta} \subset G$ is a maximal torus), respectively.

Denote by $\mathcal{G}, \mathcal{T}$, and $\mathcal{L}$ the Lie algebras of the algebraic groups $G, T$, and $L$.

Let $E$ be a local non-Archimedean field of characteristic zero, $\bar{E}$ a fixed algebraic closure of $E$, and $E^{\text {nr }}$ a maximal unramified extension of $E$ in $\bar{E}$.

For a reductive group $G$ (resp. its Lie algebra $\mathcal{G}$ ) over $E$, we denote by $\mathcal{S}(G(E)$ ) (resp. $\mathcal{S}(\mathcal{G}(E))$ ) the space of locally constant measures with compact support. We denote by $\mathcal{D}(G(E)$ ) (resp. $\mathcal{D}(\mathcal{G}(E))$ ) the space of invariant distributions on $G(E)$ (resp. $\mathcal{G}(E)$ ), namely $G(E)$-invariant linear functionals on $\mathcal{S}(G(E)$ ) (resp. $\mathcal{S}(\mathcal{G}(E))$ ), where $G(E)$ acts by conjugation. Whenever necessary we equip $G(E)$ and $\mathcal{G}(E)$ with invariant measures, denoted by $\mu$, defined by a translation-invariant top degree differential form on $G$. We denote by $G(E)_{\text {tu }}\left(\right.$ resp. $\left.\mathcal{G}(E)_{\mathrm{tn}}\right)$ the set of topologically unipotent (resp. topologically nilpotent) elements of $G(E)$ (resp. $\mathcal{G}(E)$ ). Finally, we denote by $\operatorname{rk}(G)$ the $\operatorname{rank}$ of $G$ over $E$, and put $e(G):=(-1)^{\mathrm{rk}\left(G^{\mathrm{ad}}\right)}$. Note that our sign $e(G)$ differs from that defined by Kottwitz.

\section{Formulation of the MAIN RESUlT}

2.1. Let $L$ be a connected reductive group over $\mathbb{F}_{q}$, and $\bar{a}: \bar{T} \hookrightarrow L$ an embedding of a maximal elliptic torus of $L$. Following Deligne and Lusztig, we associate to every character $\bar{\theta}: \bar{T}\left(\mathbb{F}_{q}\right) \rightarrow \mathbb{C}^{\times}$in general position an irreducible cuspidal representation $\rho_{\bar{a}, \bar{\theta}}$ of $L\left(\mathbb{F}_{q}\right)$ (see [DL Prop. 7.4 and Thm. 8.3]).

2.2. There is an equivalence of categories $T \mapsto \bar{T}$ between tori over $E$ splitting over $E^{\text {nr }}$ and tori over $\mathbb{F}_{q}$. Every such $T$ has a canonical $\mathcal{O}$-structure.

Notation 2.3. a) Let $G$ be a reductive group over $E, T$ a torus over $E$ splitting over $E^{\text {nr }}$, and $a: T \hookrightarrow G$ an embedding of a maximal elliptic torus of $G$. Then $G$ splits over $E^{\mathrm{nr}}$, and $a(T(\mathcal{O}))$ lies in a unique parahoric subgroup $G_{a}$ of $G(E)$. Let 
$G_{a^{+}}$be the pro-unipotent radical of $G_{a}$. Then there exists a canonical reductive group $L_{a}$ over $\mathbb{F}_{q}$ with an identification $L_{a}\left(\mathbb{F}_{q}\right)=G_{a} / G_{a^{+}}$. Moreover, $a: T \hookrightarrow G$ induces an embedding $\bar{a}: \bar{T} \hookrightarrow L_{a}$ of a maximal elliptic torus of $L_{a}$.

b) Let $\theta: T(E) \rightarrow \mathbb{C}^{\times}$be a character in general position, trivial on $\operatorname{Ker}[T(\mathcal{O}) \rightarrow$ $\left.\bar{T}\left(\mathbb{F}_{q}\right)\right]$. Denote by $\bar{\theta}: \bar{T}\left(\mathbb{F}_{q}\right) \rightarrow \mathbb{C}^{\times}$the character of $\bar{T}\left(\mathbb{F}_{q}\right)$ defined by $\theta$. Then there exists a unique irreducible representation $\rho_{a, \theta}$ of $Z(G)(E) G_{a}$, whose central character is the restriction of $\theta$, extending the inflation to $G_{a}$ of the cuspidal Deligne-Lusztig representation $\rho_{\bar{a}, \bar{\theta}}$ of $L_{a}\left(\mathbb{F}_{q}\right)$. We denote by $\pi_{a, \theta}$ the induced cuspidal representation $\operatorname{Ind}_{Z(G)(E) G_{a}}^{G(E)} \rho_{a, \theta}$ of $G(E)$.

2.4. Recall (see Ko2, Thm 1.2]) that for every reductive group $G$ over $E, H^{1}(E, G)$ is canonically isomorphic to the group $\pi_{0}\left(Z(\widehat{G})^{\Gamma}\right)^{D}$ of characters of $\pi_{0}\left(Z(\widehat{G})^{\Gamma}\right)$. If $T$ is a maximal torus of $G$, we get a commutative diagram:

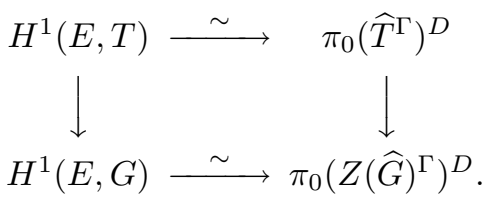

In particular, we have a canonical surjection

$$
\widehat{T}^{\Gamma} / Z(\widehat{G})^{\Gamma} \rightarrow \operatorname{Coker}\left[\pi_{0}\left(Z(\widehat{G})^{\Gamma}\right) \rightarrow \pi_{0}\left(\widehat{T}^{\Gamma}\right)\right] \stackrel{\sim}{\rightarrow}\left(\operatorname{Ker}\left[H^{1}(E, T) \rightarrow H^{1}(E, G)\right]\right)^{D} .
$$

Notation 2.5. a) To every pair $a, a^{\prime}$ of stably conjugate embeddings $T \hookrightarrow G$, one associates the class $\operatorname{inv}\left(a^{\prime}, a\right) \in \operatorname{Ker}\left[H^{1}(E, T) \rightarrow H^{1}(E, G)\right]$. This is the class of a cocycle $c_{\sigma}=g^{-1} \sigma(g)$, where $g \in G(\bar{E})$ is such that $a^{\prime}=g a g^{-1}$ (compare Ko2, 4.1]).

b) To each $\kappa \in \widehat{T}^{\Gamma} / Z(\widehat{G})^{\Gamma}$, an embedding $a_{0}: T \hookrightarrow G$, and a character $\theta$ of $T(E)$ as in Notation 2.3. we associate the invariant distribution

$$
\chi_{a_{0}, \kappa, \theta}:=e(G) \sum_{a}\left\langle\operatorname{inv}\left(a, a_{0}\right), \kappa\right\rangle \chi\left(\pi_{a, \theta}\right) .
$$

Here $a$ runs over a set of representatives of conjugacy classes of embeddings which are stably conjugate to $a_{0}$, and $\chi\left(\pi_{a, \theta}\right)$ denotes the character of $\pi_{a, \theta}$.

Notation 2.6. Each pair $(a, \kappa)$, where $a: T \hookrightarrow G$ is an embedding of a maximal torus of $G$ and $\kappa$ is an element of $\widehat{T}^{\Gamma}$, gives rise to an isomorphism class $\mathcal{E}_{(a, \kappa)}$ of an endoscopic datum of $G$. Furthermore, $\mathcal{E}_{(a, \kappa)}$ is elliptic if $a(T)$ is an elliptic torus of $G$ (see [Ko1, §7] for the definitions of endoscopic data, and compare [La1, II, 4]).

More precisely, each embedding $\eta: \widehat{T} \hookrightarrow \widehat{G}$, whose conjugacy class corresponds to the stable conjugacy class of $a$, defines an endoscopic datum $\mathcal{E}_{(a, \kappa, \eta)}=(s, \rho)$, consisting of a semisimple element $s=\eta(\kappa)$ of $\widehat{G}$ and a homomorphism $\rho: \Gamma \stackrel{\rho_{T}}{\longrightarrow}$ $\operatorname{Norm}_{\text {Aut } \widehat{G}}(\eta(\widehat{T}))_{s} / \eta(\widehat{T}) \stackrel{\rho^{\prime}}{\longrightarrow} \operatorname{Out}\left(\widehat{G}_{s}^{0}\right)$. Here $\rho_{T}$ is induced by the $E$-structure of $T$, and $\rho^{\prime}$ is induced by the inclusion $\operatorname{Norm}_{\text {Aut } \widehat{G}}(\eta(\widehat{T}))_{s} \subset \operatorname{Norm}_{\text {Aut } \widehat{G}}\left(\widehat{G}_{s}^{0}\right)$. Moreover, the isomorphism class of $\mathcal{E}_{(a, \kappa, \eta)}$, denoted by $\mathcal{E}_{(a, \kappa)}$, does not depend on $\eta$.

Notation 2.7. For each $\gamma \in G^{\mathrm{sr}}(E)$ and $\xi \in{\widehat{G_{\gamma}}}^{\Gamma}$,

(i) put $\mathcal{E}_{(\gamma, \xi)}:=\mathcal{E}_{\left(a_{\gamma}, \xi\right)}$, where $a_{\gamma}: G_{\gamma} \hookrightarrow G$ is the inclusion map; 
(ii) fix an invariant measure $d g_{\gamma}$ on $G_{\gamma}(E)$, and put

$$
O_{\gamma}(\phi):=\int_{G(E) / G_{\gamma}(E)} f\left(g \gamma g^{-1}\right) \frac{d g}{d g_{\gamma}}
$$

for each $\phi=f d g \in \mathcal{S}(G(E))$;

(iii) denote by $\bar{\xi} \in \pi_{0}\left(\widehat{G}_{\gamma}^{\Gamma} / Z(\widehat{G})^{\Gamma}\right)$ the class of $\xi$;

(iv) denote by $O_{\gamma}^{\bar{\xi}} \in \mathcal{D}(G(E))$ the sum $\sum_{\gamma^{\prime}}\left\langle\operatorname{inv}\left(\gamma^{\prime}, \gamma\right), \bar{\xi}\right\rangle O_{\gamma^{\prime}}$, taken over a set of representatives of the conjugacy classes stably conjugate to $\gamma$, where each $d g_{\gamma^{\prime}}$ is compatible with $d g_{\gamma}$.

Definition 2.8. Let $\mathcal{E}$ be an endoscopic datum of $G$.

(i) A measure $\phi \in \mathcal{S}(G(E))$ is called $\mathcal{E}$-unstable if $O_{\gamma}^{\bar{\xi}}(\phi)=0$ for all pairs $(\gamma, \xi)$ as in Notation 2.7 for which $\mathcal{E}_{(\gamma, \xi)}$ is isomorphic to $\mathcal{E}$.

(ii) A distribution $F \in \mathcal{D}(G(E))$ is called $\mathcal{E}$-stable if $F(\phi)=0$ for all $\mathcal{E}$-unstable $\phi \in \mathcal{S}(G(E))$.

Theorem 2.9. Assume that $p>\operatorname{dim} G^{\mathrm{der}}$. Then for each triple $\left(a_{0}, \kappa, \theta\right)$, the distribution $\chi_{a_{0}, \kappa, \theta}$ is $\mathcal{E}_{\left(a_{0}, \kappa\right)}$-stable.

Notation 2.10. For an endoscopic datum $\mathcal{E}=(s, \rho)$, choose a representative $\widetilde{s} \in$ $\widehat{G}^{\text {sc }}$ of $s$, and let $Z(\mathcal{E})$ be the set of $z \in Z\left(\widehat{G}^{\text {sc }}\right)^{\Gamma}$ for which there exists $g \in \widehat{G}_{s}$ commuting with $\rho: \Gamma \rightarrow \operatorname{Out}\left(\widehat{G}_{s}^{0}\right)$ such that $g \widetilde{s} g^{-1}=z \widetilde{s}$. Then $Z(\mathcal{E})$ is a subgroup of $Z\left(\widehat{G}^{\mathrm{sc}}\right)^{\Gamma}$, depending only on the isomorphism class of $\mathcal{E}$.

Definition 2.11. Let $\mathcal{E}$ be an endoscopic datum of $G$. An inner twisting $\varphi: G \rightarrow$ $G^{\prime}$ is called $\mathcal{E}$-admissible if the corresponding class $\operatorname{inv}\left(G^{\prime}, G\right) \in H^{1}\left(E, G^{\text {ad }}\right) \cong$ $\left(Z\left(\widehat{G}^{\mathrm{sc}}\right)^{\Gamma}\right)^{D}$ is orthogonal to $Z(\mathcal{E}) \subset Z\left(\widehat{G}^{\mathrm{sc}}\right)^{\Gamma}$.

Definition 2.12. Let $G$ be a reductive group over $E, \mathcal{E}=(s, \rho)$ an elliptic endoscopic datum of $G$, and $\varphi: G \rightarrow G^{\prime}$ an $\mathcal{E}$-admissible inner twisting. Fix a triple $\left(a, a^{\prime} ; \kappa\right)$, consisting of a pair $a: T \hookrightarrow G$ and $a^{\prime}: T \hookrightarrow G^{\prime}$ of stably conjugate embeddings of maximal tori, and an element $\kappa \in \widehat{T}^{\Gamma}$ such that $\mathcal{E}_{(a, \kappa)} \cong \mathcal{E}$.

a) Consider $\phi \in \mathcal{S}(G(E))$ and $\phi^{\prime} \in \mathcal{S}\left(G^{\prime}(E)\right)$. They are called $\left(a, a^{\prime} ; \kappa\right)$-indistinguishable if they satisfy the following conditions.

(A) For every $\gamma \in G^{\mathrm{sr}}(E)$ and $\xi \in{\widehat{G_{\gamma}}}^{\Gamma}$ such that $\mathcal{E}_{(\gamma, \xi)} \cong \mathcal{E}$ and $O_{\gamma}^{\bar{\xi}}(\phi) \neq 0$,

(i) there exists $\gamma^{\prime} \in G^{\prime}(E)$ stably conjugate to $\gamma$;

(ii) we have $O_{\gamma^{\prime}}^{\bar{\xi}}\left(\phi^{\prime}\right)=\left\langle\frac{\gamma^{\prime}, \gamma ; \xi}{a^{\prime}, a ; \kappa}\right\rangle O_{\gamma}^{\bar{\xi}}(\phi)$.

Here $\left\langle\frac{\gamma^{\prime}, \gamma ; \xi}{a^{\prime}, a ; \kappa}\right\rangle \in \mathbb{C}^{\times}$is the invariant $\left\langle\frac{a_{\gamma^{\prime}}, a_{\gamma} ; \xi}{a^{\prime}, a ; \kappa}\right\rangle$ defined in the Appendix for embeddings $a_{\gamma}: G_{\gamma} \hookrightarrow G$ and $a_{\gamma^{\prime}}: G_{\gamma} \hookrightarrow G^{\prime}$ such that $a_{\gamma}(\gamma)=\gamma$ and $a_{\gamma^{\prime}}(\gamma)=\gamma^{\prime}$

(B) Condition (A) holds if $G, a_{0}, \gamma, \phi$ are interchanged with $G^{\prime}, a_{0}^{\prime}, \gamma^{\prime}, \phi^{\prime}$.

b) The distributions $F \in \mathcal{D}(G(E))$ and $F^{\prime} \in \mathcal{D}\left(G^{\prime}(E)\right)$ are called $\left(a, a^{\prime} ; \kappa\right)$ equivalent if $F(\phi)=F^{\prime}\left(\phi^{\prime}\right)$ for every two $\left(a, a^{\prime} ; \kappa\right)$-indistinguishable measures $\phi$ and $\phi^{\prime}$.

Remark 2.13. If $\phi$ is $\mathcal{E}_{\left(a_{0}, \kappa\right)}$-unstable, then $\phi$ and $\phi^{\prime}=0$ are $\left(a_{0}, a_{0}^{\prime} ; \kappa\right)$-indistinguishable. Therefore every two $\left(a_{0}, a_{0}^{\prime} ; \kappa\right)$-equivalent distributions $F$ and $F^{\prime}$ are $\mathcal{E}_{\left(a_{0}, \kappa\right) \text {-stable. }}$ 
Main Theorem 2.14. Assume that $p>\operatorname{dim} G^{\mathrm{der}}$. Let $\varphi: G \rightarrow G^{\prime}$ be an $\mathcal{E}_{\left(a_{0}, \kappa\right)^{-}}$ admissible inner twisting. Let $a_{0}^{\prime}: T \hookrightarrow G^{\prime}$ be an embedding which is stably conjugate to $a_{0}$. Then the distributions $\chi_{a_{0}, \kappa, \theta}$ on $G(E)$ and $\chi_{a_{0}^{\prime}, \kappa, \theta}$ on $G^{\prime}(E)$ are $\left(a_{0}, a_{0}^{\prime} ; \kappa\right)$-equivalent.

Remark 2.15. a) By Remark 2.13, Theorem 2.9] follows from the Main Theorem.

b) We believe that a much smaller bound on $p$ would suffice.

\section{BASIC INGREDIENTS OF THE ARGUMENT}

3.1. A generalization of a theorem of Waldspurger. Suppose that we are in the situation of Definition 2.12, Then $\varphi$ induces an inner twisting $\mathcal{G} \rightarrow \mathcal{G}^{\prime}$. As in Definition 2.12 one can define $\left(a, a^{\prime} ; \kappa\right)$-equivalence of $F \in \mathcal{D}(\mathcal{G}(E))$ and $F^{\prime} \in \mathcal{D}\left(\mathcal{G}^{\prime}(E)\right)$.

Fix a nontrivial character $\psi: E \rightarrow \mathbb{C}^{\times}$, a nondegenerate $G$-invariant pairing $\langle\cdot, \cdot\rangle$ on $\mathcal{G}$, and $\varphi$-compatible invariant measures on $\mathcal{G}(E)$ and $\mathcal{G}^{\prime}(E)$. Then $\varphi$ defines a nondegenerate $G^{\prime}$-invariant pairing $\langle\cdot, \cdot\rangle^{\prime}$ on $\mathcal{G}^{\prime}$. These data determine the Fourier transforms $F \mapsto \mathcal{F}(F)$ on $\mathcal{G}(E)$ and $\mathcal{G}^{\prime}(E)$.

Theorem 3.1. The distributions $F \in \mathcal{D}(\mathcal{G}(E))$ and $F^{\prime} \in \mathcal{D}\left(\mathcal{G}^{\prime}(E)\right)$ are $\left(a, a^{\prime} ; \kappa\right)$ equivalent if and only if $e(G) \mathcal{F}(F)$ and $e\left(G^{\prime}\right) \mathcal{F}\left(F^{\prime}\right)$ are $\left(a, a^{\prime} ; \kappa\right)$-equivalent.

The proof is a generalization of that of Waldspurger [Wa], who treated the case $\phi^{\prime}=0$ (compare also [KP, Thm. 2.7.1], where the stable case is considered).

3.2. Springer hypothesis. In the notation of 2.1 assume that $\bar{a}(\overline{\mathcal{T}})\left(\mathbb{F}_{q}\right) \subset \mathcal{L}\left(\mathbb{F}_{q}\right)$ contains an $L$-regular element $\bar{t}$ [and that $p$ is so large that the logarithm defines an isomorphism log: $L_{\text {un }} \stackrel{\sim}{\rightarrow} \mathcal{L}_{\text {nil }}$ between unipotent elements of $L$ and nilpotent elements of $\mathcal{L}]$. Let $\delta_{\bar{t}}$ be the characteristic function of the $\operatorname{Ad}\left(L\left(\mathbb{F}_{q}\right)\right)$-orbit of $\bar{t}$, and let $\mathcal{F}\left(\delta_{\bar{t}}\right)$ be its Fourier transform. We need the following result of [Ka1].

Theorem 3.2. For every $u \in L_{\mathrm{un}}\left(\mathbb{F}_{q}\right)$, we have

$$
\operatorname{Tr} \rho_{\bar{a}, \bar{\theta}}(u)=q^{-(\operatorname{dim} L-\operatorname{dim} \bar{T}) / 2} \mathcal{F}\left(\delta_{\bar{t}}\right)(\log (u)) .
$$

3.3. Topological Jordan decomposition. We will call an element $\gamma \in G(E)$ compact if it generates a relatively compact subgroup of $G(E)$. We will call an element $\gamma \in G(E)$ topologically unipotent if the sequence $\left\{\gamma^{p^{n}}\right\}_{n}$ converges to 1 . Every topologically unipotent element is compact. The following result is a rather straightforward generalization of [Ka2, Lem. 2, p. 226].

Lemma 3.3. For every compact element $\gamma \in G(E)$ there exists a unique decomposition $\gamma=\delta u$ such that $\delta$ and $u$ commute, $\delta$ is of finite order prime to $p$, and $u$ is topologically unipotent. In particular, this decomposition is compatible with conjugation and field extensions.

\section{A SKetch of the PRoof of the main theorem}

\subsection{Reformulation of the problem.}

Notation 4.1. To each $a: T \hookrightarrow G$ and $\theta: T(E) \rightarrow \mathbb{C}^{\times}$as in Notation 2.3 we associate a function $t_{a, \theta}$ on $G(E)$ supported on $Z(G)(E) G_{a}$ and equal to $\operatorname{Tr} \rho_{a, \theta}$ there. Since $t_{a, \theta}$ is cuspidal, the integral

$$
F_{a, \theta}(\gamma):=\frac{1}{\mu\left(\left(G^{\mathrm{ad}}\right)_{a}\right)} \int_{G(E) / Z(G)(E)} t_{a, \theta}\left(g \gamma g^{-1}\right) d g
$$


stabilizes for every $\gamma \in G^{\mathrm{sr}}(E)$ (see [HC, Lem. 23]), thus providing us with a locally constant invariant function $F_{a, \theta}$ on $G^{\mathrm{sr}}(E)$.

Lemma 4.2. For each $a$ and $\theta, F_{a, \theta}$ is a locally $L^{1}$-function on $G(E)$. Moreover, the corresponding distribution equals $\chi\left(\pi_{a, \theta}\right)$.

Proof. The assertion follows from Harish-Chandra's theorem [HC, Thm. 16].

Notation 4.3. For every $\gamma_{0} \in G^{\mathrm{sr}}$ and $\bar{\xi} \in \pi_{0}\left({\widehat{G_{\gamma_{0}}}}^{\Gamma} / Z(\widehat{G})^{\Gamma}\right)$ we define

$$
\Sigma_{G ; \gamma_{0}, \bar{\xi}_{;} a_{0}, \kappa}:=e(G) \sum_{a} \sum_{\gamma}\left\langle\operatorname{inv}\left(a, a_{0}\right), \kappa\right\rangle\left\langle\operatorname{inv}\left(\gamma, \gamma_{0}\right), \bar{\xi}\right\rangle^{-1} F_{a, \theta}(\gamma),
$$

where $a$ and $\gamma$ run over sets of representatives of the conjugacy classes within the stable conjugacy classes of $a_{0}$ and $\gamma_{0}$, respectively.

Theorem 4.4. For all $\gamma_{0} \in G^{\mathrm{sr}}$ and $\bar{\xi} \in \pi_{0}\left({\widehat{G_{\gamma_{0}}}}^{\Gamma} / Z(\widehat{G})^{\Gamma}\right)$ such that $\Sigma_{G ; \gamma_{0}, \bar{\xi} ; a_{0}, \kappa} \neq 0$,

(i) there exists a representative $\xi \in{\widehat{G_{\gamma_{0}}}}^{\Gamma}$ of $\bar{\xi}$ such that $\mathcal{E}_{\gamma_{0}, \xi} \cong \mathcal{E}_{\left(a_{0}, \kappa\right)}$;

(ii) if $\varphi: G \rightarrow G^{\prime}$ is $\left(\mathcal{E}, a_{\gamma_{0}}, \bar{\xi}\right)$-admissible (see Definition A.1), then for every $\xi$ as in $(i)$ and every stably conjugate $\gamma_{0}^{\prime} \in G^{\prime}(E)$ of $\gamma_{0}$ we have

$$
\Sigma_{G^{\prime} ; \gamma_{0}^{\prime}, \bar{\xi} ; a_{0}^{\prime}, \kappa}=\left\langle\frac{\gamma_{0}^{\prime}, \gamma_{0} ; \xi}{a^{\prime}, a ; \kappa}\right\rangle \Sigma_{G ; \gamma_{0}, \bar{\xi} ; a_{0}, \kappa} .
$$

4.5. It follows from Lemma 4.2 that Theorem 4.4 is equivalent to the Main Theorem. Moreover, by standard arguments, Theorem 4.4 reduces to the case when the derived group of $G$ is simply connected.

4.6. From now on we will assume that $G^{\text {der }}=G^{\text {sc }}$. In particular, the centralizer of each semisimple element of $G$ is connected, and each $G_{a}$ is a maximal compact subgroup of $G(E)$. We fix $\left(\gamma_{0}, \bar{\xi}\right)$ such that $\Sigma_{G ; \gamma_{0}, \bar{\xi} ; a_{0}, \kappa} \neq 0$. Since $\Sigma_{G ; z \gamma_{0}, \bar{\xi} ; a_{0}, \kappa}=\theta(z) \Sigma_{G ; \gamma_{0}, \bar{\xi} ; a_{0}, \kappa}$ for each $z \in Z(G)(E)$ and since the support of each $t_{a, \theta}$ consists of elements compact modulo center, we can assume that $\gamma_{0}$ is compact with topological Jordan decomposition $\gamma_{0}=\delta_{0} u_{0}$. Moreover, we can assume that either $\gamma_{0}$ is topologically unipotent, or $\delta_{0} \notin Z(G)(E)$.

\subsection{The topologically unipotent case.}

4.7. Since $p$ does not divide the order of $Z\left(G^{\text {der }}\right)$, the canonical map $G^{\mathrm{der}}(E)_{\mathrm{tu}} \times$ $Z(G)(E)_{\mathrm{tu}} \rightarrow G(E)_{\mathrm{tu}}$ is an isomorphism. Therefore to prove Theorem 4.4 for topologically unipotent $\gamma_{0}$, we can assume that $G$ is semisimple and simply connected.

Notation 4.8. Denote by $\Phi_{G}: G \rightarrow \mathcal{G}$ the composition map

$$
G \stackrel{\operatorname{Ad}}{\longrightarrow} G L(\mathcal{G}) \stackrel{\log _{(p)}}{\longrightarrow} \operatorname{End}(\mathcal{G}) \stackrel{\mathrm{pr}}{\longrightarrow} \mathcal{G},
$$

where $\log _{(p)}(1-A)=-\sum_{i=1}^{p-1} \frac{A^{i}}{i}$, and pr is the canonical projection, defined by the standard pairing $(A, B) \mapsto \operatorname{Tr} A B$ on $\operatorname{End}(\mathcal{G})$.

Lemma 4.9. The map $\Phi_{G}$ defines a $G(E)$-equivariant homeomorphism

$$
G(E)_{\mathrm{tu}} \stackrel{\sim}{\rightarrow} \mathcal{G}(E)_{\mathrm{tn}}
$$

where $G(E)$ acts by conjugation. Moreover, for every parahoric subgroup $G_{x}$ of $G(E), \Phi_{G}$ induces a bijection $\left(\Phi_{G}\right)_{x}:\left(G_{x}\right)_{\mathrm{tu}} \stackrel{\sim}{\rightarrow}\left(\mathcal{G}_{x}\right)_{\mathrm{tn}}$, which in turn induces the logarithm map $\log :\left(L_{x}\right)_{\mathrm{un}}\left(\mathbb{F}_{q}\right) \stackrel{\sim}{\rightarrow}\left(\mathcal{L}_{x}\right)_{\text {nil }}\left(\mathbb{F}_{q}\right)$. 
Notation 4.10. a) By our assumption on $p$, there exists $t \in \mathcal{T}(\mathcal{O})$ whose reduction $\bar{t} \in \overline{\mathcal{T}}\left(\mathbb{F}_{q}\right)$ is not fixed by any nontrivial element of the Weyl group of $G$.

b) For every $a: T \hookrightarrow G$ as in Notation 2.3 we denote by $\Omega_{a, t} \subset \mathcal{L}_{a}\left(\mathbb{F}_{q}\right)$ the $\operatorname{Ad}\left(L_{a}\left(\mathbb{F}_{q}\right)\right)$-orbit of $\bar{a}(\bar{t})$, by $\widetilde{\Omega}_{a, t} \subset \mathcal{G}_{a} \subset \mathcal{G}$ the preimage of $\Omega_{a, t}$, and let $\delta_{a, t}$ be the characteristic function of $\widetilde{\Omega}_{a, t}$.

c) As the centralizer $G_{y}$ of each $y \in \widetilde{\Omega}_{a, t}$ is $G_{a}$-conjugate to $a(T)$, the integral

$$
\Delta_{a, t}(x):=\frac{1}{\mu\left(G_{a}\right)} \int_{G(E)} \delta_{a, t}(\operatorname{Ad}(g) x) d g
$$

converges absolutely for each $x \in \mathcal{G}(E)$. Thus it defines an element of $\mathcal{D}(\mathcal{G}(E))$. Similarly to Notation 2.5, we consider $\Delta_{a_{0}, \kappa, t}:=e(G) \sum_{a}\left\langle\operatorname{inv}\left(a, a_{0}\right), \kappa\right\rangle \Delta_{a, t} \in$ $\mathcal{D}(\mathcal{G}(E))$.

Lemma 4.11. Let $\mathcal{I}^{+} \subset \mathcal{G}(E)$ be a maximal topologically nilpotent subalgebra. Assume that $\psi: E \rightarrow \mathbb{C}^{\times}$is trivial on the maximal ideal $M \subset \mathcal{O}$ and induces a nontrivial character of $\mathbb{F}_{q}$. Then for each $u \in G(E)_{\mathrm{tu}}$ we have

$$
t_{a, \theta}(u)=\mu\left(\mathcal{I}^{+}\right)^{-1} \mathcal{F}\left(\delta_{a, t}\right)\left(\Phi_{G}(u)\right) .
$$

Proof. The assumption on $\psi$ implies that $\mathcal{G}_{a^{+}}$is the orthogonal complement of $\mathcal{G}_{a}$ with respect to the pairing $(x, y) \mapsto \psi(\langle x, y\rangle)$. Therefore our lemma is an immediate consequence of the definition of the Fourier transform (over $E$ and $\mathbb{F}_{q}$ ), Theorem 3.2, Lemma 4.9, and the equality $q^{\left(\operatorname{dim} L_{a}-\operatorname{dim} \bar{T}\right) / 2} \mu\left(\mathcal{G}_{a^{+}}\right)=\mu\left(\mathcal{I}^{+}\right)$.

4.12. Now we are ready to show that $\left(\chi_{a_{0}, \kappa, \theta}\right)_{\mid G(E)_{\text {tu }}}$ and $\left(\chi_{a_{0}^{\prime}, \kappa, \theta}\right)_{\mid G^{\prime}(E)_{\text {tu }}}$ are $\left(a_{0}, a_{0}^{\prime} ; \kappa\right)$-equivalent. First of all, by direct calculations, $e(G) \Delta_{a_{0}, \kappa, t}$ is $\left(a_{0}, a_{0}^{\prime} ; \kappa\right)$ equivalent to $e\left(G^{\prime}\right) \Delta_{a_{0}^{\prime}, \kappa, t}$. Hence, by Theorem 3.1, $\mathcal{F}\left(\Delta_{a_{0}, \kappa, t}\right)$ is $\left(a_{0}, a_{0}^{\prime} ; \kappa\right)$-equivalent to $\mathcal{F}\left(\Delta_{a_{0}^{\prime}, \kappa, t}\right)$. Using Lemmas 4.2 4.9, and 4.11 we see that $\chi_{a_{0}, \kappa, \theta}$ has the same restriction to $G(E)_{\text {tu }}$ as $\mu\left(\mathcal{I}^{+}\right)^{-1} \Phi_{G}^{*}\left(\mathcal{F}\left(\Delta_{a_{0}, \kappa, t}\right)\right)$, and similarly for $\chi_{a_{0}^{\prime}, \kappa, \theta}$ and $\mu\left(\mathcal{I}^{\prime+}\right)^{-1} \Phi_{G^{\prime}}^{*}\left(\mathcal{F}\left(\Delta_{a_{0}^{\prime}, \kappa, t}\right)\right)$. Since $\Phi_{G}$ is a $G^{\text {ad }}$-invariant algebraic morphism defined over $E$, the assertion follows from the equality $\mu\left(\mathcal{I}^{+}\right)=\mu\left(\mathcal{I}^{\prime+}\right)$.

4.3. The general case. It remains to prove Theorem 4.4 for $\delta_{0} \notin Z(G)(E)$ (see 4.6). We are going to deduce the assertion from that for $G_{\delta_{0}}$.

Proposition 4.13. For every embedding $a: T \hookrightarrow G$ and a compact element $\gamma$ in $G(E)$ with topological Jordan decomposition $\gamma=\delta u$, we have

$$
e(G) F_{a, \theta}(\gamma)=e\left(G_{\delta}\right) \sum_{b} \theta\left(b^{-1}(\delta)\right) F_{b, \theta}(u) .
$$

Here $b$ runs over the set of conjugacy classes of embeddings $b: T \hookrightarrow G_{\delta}$ whose composition with the inclusion $G_{\delta} \subset G$ is conjugate to a.

Proof. The proposition follows by direct calculation from the recursive formula ([DL, Thm. 4.2]) for characters of Deligne-Lusztig representations.

Notation 4.14. a) We say that $t \in T(E)$ is $\left(G, a_{0}, \gamma_{0}\right)$-relevant if there exists an embedding $b_{0}: T \hookrightarrow G_{\delta_{0}} \subset G$ stably conjugate to $a_{0}$ such that $b(t)=\delta_{0}$.

b) Assume that $t \in T(E)$ is $\left(G, a_{0}, \gamma_{0}\right)$-relevant. Since $a_{0}(T) \subset G$ is elliptic, for each $\delta \in G(E)$ stably conjugate to $\delta_{0}$ there exists an embedding $b_{t, \delta}: T \hookrightarrow G_{\delta} \subset G$ stably conjugate to $a_{0}$ such that $b_{t, \delta}(t)=\delta$. Further, $b_{t, \delta}$ is unique up to stable conjugacy, and the endoscopic datum $\mathcal{E}_{t, \kappa}:=\mathcal{E}_{\left(b_{\delta, t}, \kappa\right)}$ of $G_{\delta_{0}}$ is independent of $\delta$. 
c) We will write $\delta_{1} \sim_{\mathcal{E}_{t, \kappa}} \delta$ (resp. $\delta^{\prime} \sim_{\mathcal{E}_{t, \kappa}} \delta$ ) if $\delta, \delta_{1} \in G(E)$ (resp. $\delta \in G(E)$ and $\delta^{\prime} \in G^{\prime}(E)$ ) are stably conjugate to $\delta_{0}$, and $G_{\delta_{1}}$ (resp. $G_{\delta^{\prime}}^{\prime}$ ) is an $\mathcal{E}_{t, \kappa}$-admissible inner form of $G_{\delta}$ (see Definition [2.11).

4.15. Using Proposition 4.13 we see that

$$
\Sigma_{G ; \gamma_{0}, \bar{\xi} ; a_{0}, \kappa}=\sum_{t} \theta(t) \sum_{\delta} I_{t, \delta}
$$

where

(i) $t$ runs over the set of $\left(G, a_{0}, \gamma_{0}\right)$-relevant elements of $T(E)$;

(ii) $\delta$ runs over a set of representatives of the conjugacy classes within the stable conjugacy class of $\delta_{0}$;

(iii) $I_{t, \delta}$ vanishes unless there exists an element $\gamma \in G(E)$ stably conjugate to $\gamma_{0}$ with topological Jordan decomposition $\gamma=\delta u$, in which case we get

$$
I_{t, \delta}=\left\langle\operatorname{inv}\left(b_{t, \delta}, a_{0}\right), \kappa\right\rangle\left\langle\operatorname{inv}\left(\gamma, \gamma_{0}\right), \bar{\xi}\right\rangle^{-1} \Sigma_{G_{\delta} ; u, \bar{\xi} ; b_{t, \delta}, \kappa}
$$

4.16. For simplicity of the exposition, we will restrict ourselves to the case when $\gamma_{0} \in G(E)$ is elliptic. Choose $t$ which has a nonzero contribution to (4.2). Replacing $\delta_{0}$ by a stably conjugate element we can assume that $\sum_{\delta \sim_{\mathcal{E}_{t, \kappa}} \delta_{0}} I_{t, \delta} \neq 0$ and $I_{t, \delta_{0}} \neq$ 0 . So $\Sigma_{G_{\delta_{0}} ; u_{0}, \bar{\xi} ; b_{t, \delta}, \kappa} \neq 0$. Hence by Theorem 4.4 for $G_{\delta_{0}}$ there exists a representative $\xi \in{\widehat{G_{\gamma_{0}}}}^{\Gamma}$ of $\bar{\xi}$ such that the endoscopic datum $\mathcal{E}_{\left(u_{0}, \xi\right)}$ of $G_{\delta_{0}}$ is isomorphic to $\mathcal{E}_{t, \kappa}$. Therefore there exist embeddings $\eta_{1}: \widehat{G_{\gamma_{0}}} \hookrightarrow \widehat{G_{\delta_{0}}}$ and $\eta_{2}: \widehat{T} \hookrightarrow \widehat{G_{\delta_{0}}}$ such that $\mathcal{E}_{\left(\gamma_{0}, \xi, \eta_{1}\right)}=\mathcal{E}_{\left(b_{t, \delta_{0}}, \kappa, \eta_{2}\right)}$ (compare Notation 2.6) and $\eta_{2}(\kappa)=z \eta_{1}(\xi)$ for a certain $z \in Z\left(\widehat{G_{\delta_{0}}}\right)^{\Gamma}$. Moreover, $z$ is defined up to multiplication by an element of $Z\left(\mathcal{E}_{t, \kappa}\right)$. Therefore for all $\delta \sim_{\mathcal{E}_{t, \kappa}} \delta_{0}$, the expression $\left\langle\operatorname{inv}\left(\delta, \delta_{0}\right), z\right\rangle$ is independent of the choice of the $\eta_{i}$ 's.

Claim 4.17. For each $\delta \sim_{\mathcal{E}_{t, \kappa}} \delta_{0}$ we have $I_{t, \delta}=\left\langle\operatorname{inv}\left(\delta, \delta_{0}\right), z\right\rangle I_{t, \delta_{0}}$.

Proof. Since $\Sigma_{G_{\delta_{0}} ; u_{0}, \bar{\xi} ; b_{t, \delta}, \kappa} \neq 0$, Theorem 4.4 for inner forms $G_{\delta}$ and $G_{\delta_{0}}$ implies that for every stably conjugate $u \in G_{\delta}(E)$ of $u_{0} \in G_{\delta_{0}}(E)$, we have

$$
\Sigma_{G_{\delta} ; u, \bar{\xi} ; b_{t, \delta}, \kappa}=\left\langle\frac{u, u_{0} ; \xi}{b_{t, \delta}, b_{t, \delta_{0}} ; \kappa}\right\rangle \Sigma_{G_{\delta_{0}} ; u_{0}, \bar{\xi} ; b_{t, \delta_{0}}, \kappa} \cdot
$$

Then $\gamma:=\delta u \in G(E)$ is stably conjugate to $\gamma_{0}$, and the assertion follows by direct calculation from (4.3).

4.18. Now we are ready to show the validity of (i), (ii) of Theorem 4.4

(i) As $\sum_{\delta \sim_{\mathcal{E}_{t, \kappa}} \delta_{0}} I_{t, \delta} \neq 0$, we get from Claim 4.17 that $\sum_{\delta \sim_{\mathcal{E}_{t, \kappa}} \delta_{0}}\left\langle\operatorname{inv}\left(\delta, \delta_{0}\right), z\right\rangle \neq$ 0 . By the definition of $\mathcal{E}_{t, \kappa}$-equivalence, this implies that $z$ belongs to $Z\left(\mathcal{E}_{t, \kappa}\right) Z(\widehat{G})^{\Gamma}$. Thus changing $\eta_{1}$ (or $\eta_{2}$ ), we can assume that $z \in Z(\widehat{G})^{\Gamma}$. Since $\left(\gamma_{0}, \xi\right)$ and $\left(b_{t, \delta_{0}}, \kappa\right)$ define isomorphic endoscopic data of $G_{\delta_{0}}$, we therefore conclude that $\mathcal{E}_{\left(\gamma_{0}, \xi\right)} \cong$ $\mathcal{E}_{\left(a_{0}, \kappa\right)}$.

(ii) Since $T$ is elliptic, an element $t \in T(E)$ is $\left(G, a_{0}, \gamma_{0}\right)$-relevant if and only if it is $\left(G^{\prime}, a_{0}^{\prime}, \gamma_{0}^{\prime}\right)$-relevant. Thus it will suffice to show that for every such $t$ we have $\sum_{\delta^{\prime}} I_{t, \delta^{\prime}}=\left\langle\frac{\gamma_{0}^{\prime}, \gamma_{0} ; \xi}{a_{0}^{\prime}, a_{0} ; \kappa}\right\rangle \sum_{\delta} I_{t, \delta}$. For every stably conjugate $\delta \in G(E)$ of $\delta_{0}$, there exists a stably conjugate $\delta^{\prime} \in G^{\prime}(E)$ of $\delta_{0}^{\prime}$ such that $\delta^{\prime} \sim \mathcal{E}_{t, \kappa} \delta$. Therefore it will suffice to show that for every such pair $\delta^{\prime} \sim \mathcal{E}_{t, \kappa} \delta$, we have $I_{t, \delta^{\prime}}=\left\langle\frac{\gamma_{0}^{\prime}, \gamma_{0} ; \xi}{a_{0}^{\prime}, a_{0} ; \kappa}\right\rangle I_{t, \delta}$. The latter equality can be proved by the same arguments as Claim 4.17 


\section{APPENDix A.}

Let $G$ be a reductive group over $E, \mathcal{E}=(s, \rho)$ an elliptic endoscopic datum of $G$, and $\varphi: G \rightarrow G^{\prime}$ an $\mathcal{E}$-admissible inner twisting. For every two triples $\left(a_{i}^{\prime}, a_{i} ; \kappa_{i}\right)$, $i=1,2$, where $a_{i}: T_{i} \hookrightarrow G$ and $a_{i}^{\prime}: T_{i} \hookrightarrow G^{\prime}$ are stably conjugate embeddings of maximal tori, and $\kappa_{i}$ is an element of $\widehat{T}_{i}^{\Gamma}$ such that $\mathcal{E}_{\left(a_{i}, \kappa_{i}\right)}$ is isomorphic to $\mathcal{E}$, we are going to define an invariant $\left\langle\frac{a_{1}^{\prime}, a_{1} ; \kappa_{1}}{a_{2}^{\prime}, a_{2} ; \kappa_{2}}\right\rangle \in \mathbb{C}^{\times}$.

Step 1. Replacing $G, G^{\prime}, T_{i}, \kappa_{i}$, and $\mathcal{E}$ by $G^{\mathrm{sc}}, G^{\prime \mathrm{sc}}, T_{i}^{\mathrm{sc}}:=a_{i}^{-1}\left(G^{\mathrm{sc}}\right)=a_{i}^{\prime-1}\left(G^{\prime \mathrm{sc}}\right)$, the image of $\kappa_{i}$ in $\widehat{T_{i}^{\mathrm{sc}}} \Gamma$, and the corresponding endoscopic datum of $G^{\mathrm{sc}}$, respectively, we can assume that $G$ is semisimple and simply connected. Let $T_{1,2}$ be the quotient of the product $T_{1} \times T_{2}$ by the subgroup $\left\{\left(z, z^{-1}\right) \mid z \in Z(G)=Z\left(G^{\prime}\right)\right\}$.

Step 2. Choose elements $g_{1}, g_{2}$, and $\left\{\widetilde{c}_{\sigma}\right\}_{\sigma \in \Gamma}$ of $G(\bar{E})$ such that $a_{i}^{\prime}=\varphi\left(g_{i} a_{i} g_{i}^{-1}\right)$ and each $\widetilde{c}_{\sigma}$ is a representative of $\varphi^{-1 \sigma} \varphi \in G^{\text {ad }}(\bar{E})$. Then each $g_{i}^{-1} \widetilde{c}_{\sigma}{ }^{\sigma} g_{i} \in G(\bar{E})$ belongs to $a_{i}\left(T_{i}(\bar{E})\right)$, and the images of $\left(a_{1}^{-1}\left(g_{1}^{-1} \widetilde{c}_{\sigma}^{\sigma} g_{1}\right), a_{2}^{-1}\left(\left(g_{2}^{-1} \widetilde{c}_{\sigma}{ }^{\sigma} g_{2}\right)^{-1}\right)\right)$ in $T_{1,2}(\bar{E})$ form a cocycle, whose cohomology class $\operatorname{inv}\left(\frac{a_{1}^{\prime}, a_{1}}{a_{2}^{\prime}, a_{2}}\right) \in H^{1}\left(E, T_{1,2}\right)$ is independent of the choices.

Step 3. Choose embeddings $\eta_{i}: \widehat{T}_{i} \hookrightarrow \widehat{G}$ such that $\mathcal{E}_{\left(a_{i}, \kappa_{i}, \eta_{i}\right)}=(s, \rho)$ and a representative $\widetilde{s} \in \widehat{G}^{\text {sc }}=\widehat{G^{\text {ad }}}$ of $s$. Put $T_{i}^{\text {ad }}:=T_{i} / a_{i}^{-1}(Z(G))$. Each $\eta_{i}$ defines an embedding $\widetilde{\eta}_{i}: \widehat{T_{i}^{\text {ad }}} \hookrightarrow \widehat{G^{\text {ad }}}$, hence an element $\widetilde{\kappa}_{i}=\kappa\left(\widetilde{s}, \eta_{i}\right):=\widetilde{\eta}_{i}^{-1}(\widetilde{s}) \in \widehat{T_{i}^{\text {ad }}}$. Then the image of $\left(\widetilde{\kappa}_{1}, \widetilde{\kappa}_{2}\right)$ in $\widehat{T_{1}^{\text {ad }}} \times \widehat{T_{2}^{\text {ad }}} / Z\left(\widehat{G^{\text {ad }}}\right) \cong \widehat{T_{1,2}}$, denoted by $\kappa_{1,2}$, is $\Gamma$ invariant. Moreover, as $\varphi: G \rightarrow G^{\prime}$ is $\mathcal{E}$-admissible, the expression $\left\langle\frac{a_{1}^{\prime}, a_{1} ; \kappa_{1}}{a_{2}^{\prime}, a_{2} ; \kappa_{2}}\right\rangle:=$ $\left\langle\operatorname{inv}\left(\frac{a_{1}^{\prime}, a_{1}}{a_{2}^{\prime}, a_{2}}\right), \kappa_{1,2}\right\rangle \in \mathbb{C}^{\times}$is independent of the choices.

Definition A.1. Let $\mathcal{E}=(s, \rho)$ be an endoscopic datum of $G, \varphi: G \rightarrow G^{\prime}$ an inner twisting, $a: T \hookrightarrow G$ an embedding of a maximal torus, and $\kappa$ an element of $\widehat{T}^{\Gamma}$ such that $\mathcal{E}_{(a, \kappa)} \cong \mathcal{E}$. We say that $\varphi: G \rightarrow G^{\prime}$ is $(\mathcal{E}, a, \bar{\kappa})$-admissible, if for all representatives $\kappa^{\prime} \in \widehat{T}^{\Gamma}$ of $\bar{\kappa} \in \pi_{0}\left(\widehat{T}^{\Gamma} / Z(\widehat{G})^{\Gamma}\right)$ satisfying $\mathcal{E}_{\left(a, \kappa^{\prime}\right)} \cong \mathcal{E}$, all embeddings $\eta, \eta^{\prime}: \widetilde{T} \hookrightarrow \widehat{G}$ such that $\mathcal{E}_{(a, \kappa, \eta)}=\mathcal{E}_{\left(a, \kappa^{\prime}, \eta^{\prime}\right)}=(s, \rho)$, and all representatives $\widetilde{s} \in$ $\widehat{G^{\text {ad }}}$ of $s$, the difference $\kappa\left(\widetilde{s}, \eta^{\prime}\right)-\kappa(\widetilde{s}, \eta) \in Z\left(\widehat{G^{\text {ad }}}\right)^{\Gamma}$ is orthogonal to $\operatorname{inv}\left(G^{\prime}, G\right) \in$ $H^{1}\left(E, G^{\text {ad }}\right)$.

Remark A.2. a) Every $(\mathcal{E}, a, \bar{\kappa})$-admissible inner twisting is $\mathcal{E}$-admissible.

b) If $a(T) \subset G$ is elliptic, then every $\mathcal{E}$-admissible inner twisting is $(\mathcal{E}, a, \bar{\kappa})$ admissible.

c) $\varphi: G \rightarrow G^{\prime}$ is $\left(\mathcal{E}, a_{1}, \bar{\kappa}_{1}\right)$-admissible if and only if $\left\langle\frac{a_{1}^{\prime}, a_{1} ; \kappa_{1}}{a_{2}^{\prime}, a_{2} ; \kappa_{2}}\right\rangle=\left\langle\frac{a_{1}^{\prime}, a_{1} ; \kappa_{1}^{\prime}}{a_{2}^{\prime}, a_{2} ; \kappa_{2}}\right\rangle$ for all representatives $\kappa_{1}^{\prime} \in \widehat{T}_{1}^{\Gamma}$ of $\bar{\kappa}_{1} \in \pi_{0}\left(\widehat{T}_{1}^{\Gamma} / Z(\widehat{G})^{\Gamma}\right)$ satisfying $\mathcal{E}_{\left(a_{1}, \kappa_{1}^{\prime}\right)} \cong \mathcal{E}$.

\section{REFERENCES}

[De] P. Deligne, Les corps locaux de caractéristique p, limites de corps locaux de caractéristique 0, in Représentations des groupes réductifs sur un corps local, pp. 119-157, Hermann, Paris, 1984. MR 86g:11068

[DL] P. Deligne and G. Lusztig, Representations of reductive groups over finite fields, Ann. of Math. (2) 103 (1976), 103-161. MR 52:14076

[HC] Harish-Chandra, Harmonic analysis on reductive p-adic groups (Notes by G. van Dijk), Lecture Notes in Mathematics 162, Springer-Verlag, Berlin-New York, 1970. MR 54:2889 
[Ka1] D. Kazhdan, Proof of Springer's hypothesis, Israel J. Math. 28 (1977), 272-286. MR 58:5959

[Ka2] D. Kazhdan, On lifting, in Lie group representations, II (College Park, Md., 1982/1983), 209-249, Lecture Notes in Mathematics 1041, Springer, Berlin, 1984. MR 86h:22029

[Ka3] D. Kazhdan, Representations of groups over close local fields, J. Analyse Math. 47 (1986), 175-179. MR 88g:22018

[KP] D. Kazhdan and A. Polishchuk, Generalization of a theorem of Waldspurger to nice representations, in The orbit method in geometry and physics (Marseille, 2000), 197-242, Progr. Math. 213, Birkhäuser, Boston, 2003.

[Ko1] R. E. Kottwitz, Stable trace formula: cuspidal tempered terms, Duke Math. J. 51 (1984), 611-650. MR 85m:11080

[Ko2] R. E. Kottwitz, Stable trace formula: elliptic singular terms, Math. Ann. 275 (1986), 365-399. MR 88d:22027

[La1] R. P. Langlands, Les débuts d'une formule des traces stable, Publ. Math. Univ. Paris VII 13, Paris, 1983. MR 85d:11058

[La2] R. P. Langlands, Representations of abelian algebraic groups, Pacific J. Math. 1997, Special Issue, 231-250. MR 99b:11125

[Wa] J.-L., Waldspurger, Transformation de Fourier et endoscopie, J. Lie Theory 10 (2000), 195-206. MR 2001g:11078

Institute of Mathematics, Hebrew University, Givat-Ram, Jerusalem, 91904 Israel

E-mail address: kazhdan@math.huji.ac.il

Institute of Mathematics, Hebrew University, Givat-Ram, Jerusalem, 91904 Israel

E-mail address: vyakov@math.huji.ac.il 